\title{
Mechatronical system used for the recovery of motor functions
}

\author{
Roxana Andreiaș ${ }^{1, *}$, and Daniela Mariana Barbu $^{1}$ \\ ${ }^{1}$ Design Product, Mechatronics and Environment Department, Transilvania University of Brasov, Brasov, Romania
}

\begin{abstract}
Robotics of medical recovery represent a subject of great interest among various research centers that are trying to develop different complex systems dedicated to the recovery of motor functions through fulfilling the required tasks imposed by applied therapy programs. In order to implement the concept of 'robotical therapy' it is required to use a robotic system specially designed to adhere to medical protocols and to meet the requirements of therapeutic exercises by programming the command and control unit. This paper is in the field of mechatronic systems used in medicine because the purpose of this kind of equipment is to help patients who underwent surgery in the lower limbs regain the mobility of the affected joints as soon as possible. The proposed objective is to improve an exising concept so that a patient may be able to use it in the comfort of their own house, but still being $24 / 7$ monitorized using various sensors and even being able to contact medical care assistance in case of emergency.
\end{abstract}

\section{Introduction}

Rehabilitation robotics is a field of research dedicated to understanding and augmenting rehabilitation through the application of robotic devices. Rehabilitation robotics includes development of mechatronic systems specially created for assisting different sensorimotor functions(e.g. arm[1], hand[2], leg[3], ankle[4]), development of different schemes of assisting therapeutic training and assessment of sensorimotor performance (ability to move) of patient.[5]

The main objective is to use robots as therapy aids instead of assistive devices. Rehabilitation using robotics is generally well tolerated by patients and has been found to be an effective adjunct to therapy in individuals suffering from motor impairments, especially due to stroke or after surgery recovery.

Although popular culture presents the image of a robot as a mechanical, humanoid device, often evil in intent, in attempt to follow the development of rehabilitation robotics, it should all start from an official definition. The Robot Institute of America defined a robot as

„A reprogrammable, multifunctional manipulator designed to move material, parts, tools or specialized devices through variable programmed motions for the performance of a variety of tasks. "[6]

It is obvious that this definition was intended for industrial robots but it identifies the key features of programmability, flexibility and movement.

In 1987 the Department of Trade and Industry in the UK had an initiative in encouraging the usage of robots in areas other than the industrial field. Using the definition of Advanced Robotics

„, The integration of enabling technologies and attributes embracing manipulators, mobility, sensors, computing (IKBS, AI) and hierarchical control to result ultimately in a robot capable of autonomously complementing man's endeavours in unstructured and hostile environments." [6], it can be stated that the aim of the integration of advanced technologies is to develop a device that can both operate autonomously and in an enviroment that may be unstructured and/or hostile.

The use of robots in rehabilitation is often associated with assistive devices, some used in special dedicated areas, some as aids to daily living. There are some other areas where robotic technology has been applied such as in mobility, where traditional wheelchairs, prosthetics and orthotics are being developed to increase the usefulness. A major area of concern is represented by the robot mediated therapy, where robots are used to increase the effectiveness of traditional therapies and even to develop new therapy protocols.

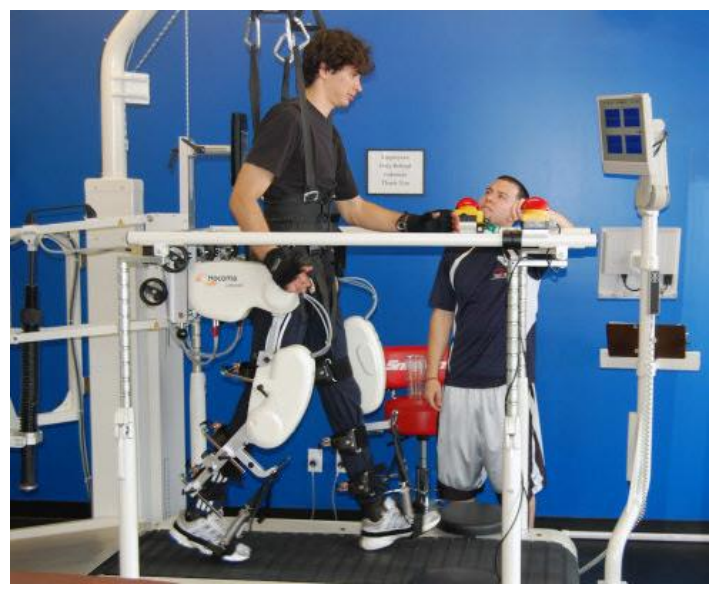

Fig. 1 - Lokomat therapy robot [7] Researches in neuroscience had shown that the brain and spinal cord retain a remarkable ability to adapt, even after injury, through the use of practiced movements. The first

\footnotetext{
* Corresponding author: andreias.roxana@yahoo.com
} 
robot used in that way, MIT-Manus [8], helped stroke patients to reach across a tabletop if they were unable to perform the task by themselves. Patients who received extra therapy from the robot improved the rate of their arm movement recovery. Another therapy robot, the Lokomat (presented in figure 1), supports the weight of a person and moves the legs in a walking pattern over a moving treadmill, with the goal of retraining the person to walk after spinal cord injury or stroke.[9]

The major limiting factor in the development of rehabilitation robots is that researchers do not know what exactly needs to happen in order for the nervous system to adapt to overcome a physical impairment. Researchers are developing rehabilitation robots that assist in movement, resist movement when it is uncoordinated, or even make movements more uncoordinated in an attempt to trick the nervous system into adapting. Advances have been made in the development of robotic exoskeletons, which are lightweight wearable devices that assist in limb movement. Other types of rehabilitation robots could play a role in assisting the nervous system to regenerate appropriate neural connections following stem cell and other medical treatments.[10]

Robotic systems have the ability to take precise measurements of kinematic and dynamic values, which are far more reliable than those achieved with human error, and have the potential to be very useful for assessment purposes. This being said, there still remains a need to develop standardized procedures and protocols in order for this data to be useful. Currently some examples of the data used from the robotics systems during assessments are ROM, walking distance, gait velocity and other various dynamic measures, but we do not yet have a standardized measure for assessment, like those seen in other gait related assessments (Barthel Index, Dynamic Gait Index, etc.).

Furthermore, the effectiveness of robotics has not been shown to be highly superior to that of typical manuallyassisted therapy provided by therapists and this is a driving reason as to why it has not yet been implemented into regular practice.[11]

One of the less obvious examples of incorporating robotics into rehabilitation is that of its assessment capabilities. While machines and receptors have been used for years in the rehab field (eg. EMG, force plates), their primary purpose has been to just collect raw data. With the right robotics, measures such as joint angles, velocities, amplitudes can be observed and recorded easily. This information can then be used as an outcome measure to see the progress a patient can make during a treatment plan. [12]

The purpose of this work is to develop a mechatronic system that will serve as an aid for patients who underwent surgery in the lower limbs in order to regain the mobility of the affected joints as soon as possible.

\section{AID device}

Gait training represents one of the main purposes for lower limb robotic rehabilitation. The current methods of improving gait require the assistance of multiple therapists to help the patient move each joint and leg appropriately for best results. This represents an expensive and very difficult approach for rehab because it is extremely labour intensive for the therapists to provide such care. [12]

Using robotics, the patient will be strapped onto the robotic device, allowing the therapists attention to be concerned only for providing supervision and for setting up of the device. This type of therapy should deliver the same mechanical movements as the traditional approach in a shorter period of time.

\subsection{CPM Machine}

Studies suggest that continuous passive motion (CPM) can improve range of motion (ROM) in those patients undergoing surgical release of arthrofibrosis of the knee or manipulation of the knee under anesthesia. In these settings, CPM provides for early post-operative motion and is considered a substitute for active physical therapy (PT).[13]

The method of rehabilitation with the use of the CPM device affects the acceleration of the process of postoperative knee rehabilitation after arthroscopy and arthroplasty.It allows, among others, the improvement of metabolism within the joint, accelerates healing of damaged parts of the joint and reduces increased soft tissue tension. Furthermore, one can observe a faster absorption of hematoma, a better blood supply to the limb and increased strength of the ligament tension.[14]

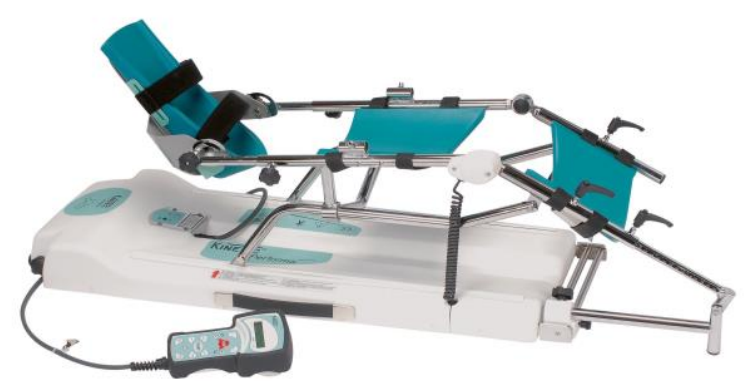

Fig. 2 - Commercial example of CPM machine (Kinetec Performa knee CPM) [15]

Figure21 presents an existing CPM machine for the purpose of studying its characteristics in order to adapt and develop an improved version.

Criteria usually considered in the design and construction of these machines are as follows:

- Adjustability to a wide range of patient sizes;

- Comfort, particularly for long uninterrupted application;

- Use in a variety of settings: in bed while sleeping, while ambulating during the day, at home or in hospital;

- Durability;

- Light weight;

- Low cost;

- Full anatomic range of motion capability; 
- Multi-axis motion capability;

- Minimum loading of joints;

- Precise and variable range of motion (ROM) control;

- Speed control;

- Safe and reliable operation;

- Simple and straightforward operation;

- Protection for unstable joints.

There is also a significant creative and innovative element to the process that can not be ignored. The art of creative product design lies precisely in being able to find simple and creative solutions to constantly broaden and redefine the range of what is considered possible.[16]

\subsection{Overview of Device}

The proposed device should be able to provide different additional functionality beyond ensuring the proper movement of the rehabilitation limb.

Mechanical components used to elaborate this device should be rigid enough to resist working under maximum load (min. $90 \mathrm{~kg}$ or greater for adult men). Also, the materials from which this device is to be made are lightweight, so the final resulted equipment could be portable and easy to manipulate. The most important aspect is that this device will meet all the safety requirements (it is to be used by patients by themselves at home).

It is designed as a combined mechanism, with an assembly of three links made from aluminum (Figure 3 : (a) Femural link ; (b) Tibial link ; (c) Plantar link) which will sustain the patient's leg that will be strapped onto the device. The femural and tibial links will be conjoined using a mobile joint (figure 3-(d)) in order to execute motions of flexion and extension of the affected knee joint. There are also four links attached to stabilize the whole process (one on each side of the assembly).

The elaborated design will also incorporate two $12 \mathrm{~V}$ servo motors (figure 3-(e)) that will ensure the movements of the linkage mechanism along the base board.

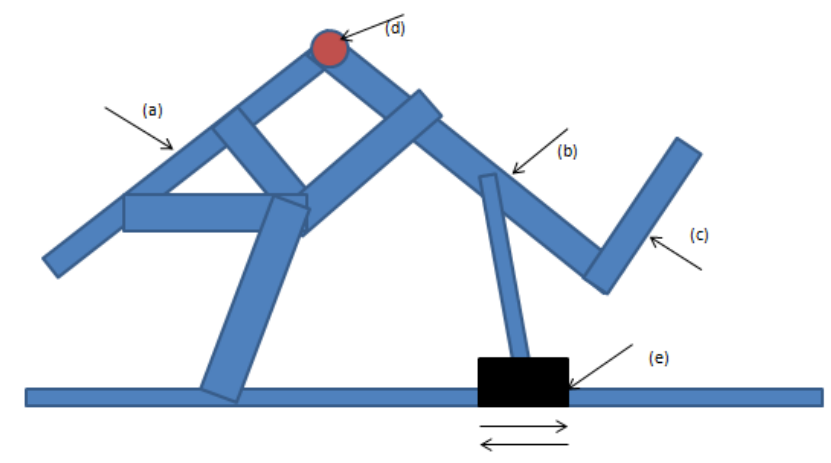

Fig. 3 - Proposed structure of own CPM machine

The device has open-loop control with a constant power supply. The outer current of the power supply can adjust the speed of the device, so that the user can control and adapt the needed motion.

This device will be adjustable for several leg lengths because the plantar link will be provided with the possibility of extension for a range of 10 to 20 centimeters.
Also, it will incorporate several soft covers for all the metallic components that come in contact with the patient's leg in order to make any procedure as comfortable as possible. Nevertheless, a fixed foot ankle orthesis can be added for more confort and better support.

\subsection{Software and Programming}

The micro controller used for the custom CPM device is an ATmega2560 Arduino board. Using the Arduino dedicated application, the source-code containing all the parameters involved and controlled during therapy programs can be uploaded and runed in order to set the motors in motion.

The application allows to upload several programs and to access any of it before or during the procedure. It will allow to adjust the amplitude of the movement, the angles needed for the knee flexion motion, the total duration of a procedure etc.

As an additional feature, several sensors can be added to the Arduino board in order to monitor various functions during the therapy procedures. Adding two-three muscular sensors will give the superviser/user of this device the possibility to monitor the muscular activity and even to avoid any overstressing of the affected joint.

\section{Results and Conclusions}

The purpose of this project is to try and create a CPM custom machine that can be used to complete a rehabilitation routine on patients that are recovering after knee surgery using mechatronic knowledge and minimal medical information.

Continuous passive motion is a common rehabilitation therapy for post-operative knee surgery patients. However, speed, duration, maximum flexion angle, and maximum extension angle have no standard settings for physical therapy methodology. Adjusting these input parameters to maximize the benefits of the post-operative rehabilitation treatment may reduce the treatment needed time.

The study or observation of the biomechanical properties of a knee undergoing passive motion can be beneficial for several surgical procedures in addition to post-operative applications. For example, this testing device will be used in our university's institute by other fellow researchers to simulate and investivate the effects of different parameter values and motion programs.

Also, we can observe passive motion for various types of prosthetics and study the effects on the biomechanical properties in the knee.

In addition to the study of the passive motion effects on the knee, this project may lead to future studies involving active motion. Passive motion in the knee does not require muscle activation.

Simulation of muscle contraction during knee flexion and extension by pulling on the tendons may give further insight into the biomechanical properties of a knee undergoing active motion, which is important in 
determining biomechanical properties during everyday activities such as walking, running, or stair climbing.

The experimental test setup will motivate further research studies that may improve the surgical techniques, reduce the treatment time for physical therapy, or improve the outcome of rehabilitation programs for post-operative knee surgery patients.

\section{References}

1. R.B. Brewer, S.K. McDowell., L.C. WorthenChaudhari, Poststroke Upper Extremity Rehabilitation: A Review of Robotic Systems and Clinical Results (Topics in Stroke Rehabilitation. 14 (6): 22-44, 2007)

2. S. Balasubramanian, J. Klein, E. Burdet, Robotassisted rehabilitation of hand function (Current Opinion in Neurology. 23, 2010)

3. Y. Kang, D. Jeon, Rehabilitation robot control using the VSD method (System Integration(SII) IEEE/SICE International Symposium: 192., 2012)

4. K.P. Michmizos, S. Rossi, E. Castelli, P. Cappa, H.I. Krebs, Robot-Aided Neurorehabilitation: A Pediatric Robot for Ankle Rehabilitation (". IEEE Transactions on Neural Systems and Rehabilitation Engineering. 23 (6): 1056-67, 2015)

5. L. Marchal-Crespo, D.J. Reinkensmeyer, Review of control strategies for robotic movement training after neurologic injury (Journal of NeuroEngineering and Rehabilitation. 6: 20, 2009)

6. S. Balasubramanian, R. Colombo, I. Sterpi, V. Sanguineti, E. Burdet, Robotic Assessment of Upper Limb Motor Function After Stroke (American Journal of Physical Medicine \& Rehabilitation. 91 (11 Suppl 3): S255-69., 2012)

7. Center For Neuro Recovery. Lokomat Pro Gait Training (20 April 2018)http://www.centerforneurorecovery.com/wpcontent/uploads/2015/06/Lokomat-Pro-RoboticGait-Training
8. H. Krebs, M. Ferraro, P.S. Buerger, M.J. Newbery, A. Makiyama, M. Sandmann, D. Lynch, B.T. Volpe, N. Hogan, Rehabilitation robotics: pilot trial of a spatial extension for MIT-Manus (Journal of NeuroEngineering and Rehabilitation. 1 (1): 5., 2004)

9. R. Riener, M. Frey, M. Bernhardt, T. Nef, G. Colombo, Human-Centered Rehabilitation Robotics (International Conference On Rehabilitation Robotics (ICORR), 2005)

10. Rehabilitation robot (20 April 2018) https://www.britannica.com/technology/rehabilitatio n-robot

11. I. Diaz, J. Gil, E. Sanches, Limb Robotic Rehabilitation: Literature Review and Challenges (J Robotics.;2011:1-11., 2011)

12. S. Maggioni, A. Melendez-Calderon, E. van Asseldonk, V. Klamroth-Marganska, L. Lünenburger, R. Riener et al., Robot-aided assessment of lower extremity functions: a review (. J Neuroeng Rehab;13(1)., 2016)

13. M. Hillman, Rehabilitation Robotics from Past to Present - A Historical Perspective (Advances in Rehabilitation Robotics, LNCIS 306, pp. 25-44, 2004)

14. R. Trochimczuk, T. Kuźmierowski, Kinematic analysis of CPM machine supporting to rehabilitation process after surgical knee arthroscopy and arthroplasty (Int. J. of Applied Mechanics and Engineering, vol.19, No.4, pp.841$848,2014)$

15. Kinetec Performa knee CPM machine (20 April 2018) http://www.thelivingcentre.co.uk/kinetecperforma.html

16. J. Saringer, Bridging theory with practice: practical considerations in the design of CPM machines (Continuous Passive Motion: A Biological Concept for the Healing and Regeneration of Articular Cartilage, Ligaments, and Tendons : From Origination to Research to Clinical, 1992) 